PROCEEDINGS OF THE

AMERICAN MATHEMATICAL SOCIETY

Volume 133, Number 7, Pages 1977-1984

S 0002-9939(05)07964-5

Article electronically published on February 24, 2005

\title{
A NOTE ON HARDY-TYPE INEQUALITIES
}

\author{
PENG GAO
}

(Communicated by Jonathan M. Borwein)

\begin{abstract}
We use a theorem of Cartlidge and the technique of Redheffer's "recurrent inequalities" to give some results on inequalities related to Hardy's inequality.
\end{abstract}

\section{INTRODUCTION}

Suppose throughout that $p \neq 0, \frac{1}{p}+\frac{1}{q}=1$. Let $l^{p}$ be the Banach space of all complex sequences $\mathbf{a}=\left(a_{n}\right)_{n \geq 1}$ with norm

$$
\|\mathbf{a}\|:=\left(\sum_{n=1}^{\infty}\left|a_{n}\right|^{p}\right)^{1 / p}<\infty .
$$

The celebrated Hardy's inequality ([11, Theorem 326) asserts that for $p>1$,

$$
\sum_{n=1}^{\infty}\left|\frac{1}{n} \sum_{k=1}^{n} a_{k}\right|^{p} \leq\left(\frac{p}{p-1}\right)^{p} \sum_{k=1}^{\infty}\left|a_{k}\right|^{p} .
$$

Among the many papers that have appeared providing new proofs, generalizations and sharpenings of (1.1), we refer the reader to the work of G. Bennett [2]- 6 ] for his study of factorable matrices.

Hardy's inequality can be regarded as a special case of the following inequality:

$$
\sum_{j=1}^{\infty}\left|\sum_{k=1}^{\infty} c_{j, k} a_{k}\right|^{p} \leq U \sum_{k=1}^{\infty}\left|a_{k}\right|^{p}
$$

in which $C=\left(c_{j, k}\right)$ and the parameter $p$ are assumed fixed $(p>1)$, and the estimate is to hold for all real sequences a. The $l^{p}$ operator norm of $C$ is then defined as the $p$-th root of the smallest value of the constant $U$ :

$$
\|C\|_{p, p}=U^{\frac{1}{p}} .
$$

Hardy's inequality thus asserts that the Cesáro matrix operator $C$, given by $c_{j, k}=1 / j, k \leq j$, and 0 otherwise, is bounded on $l^{p}$ and has norm $\leq p /(p-1)$. (The norm is in fact $p /(p-1)$.)

Received by the editors June 20, 2003.

2000 Mathematics Subject Classification. Primary 26D15, 26D20.

Key words and phrases. Hardy's inequality, Carleman's inequality, recurrent inequality.

(C)2005 American Mathematical Society 
We say a matrix $A$ is a summability matrix if its entries satisfy $a_{j, k} \geq 0, a_{j, k}=0$ for $k>j$ and $\sum_{k=1}^{j} a_{j, k}=1$. We say a summability matrix $A$ is a weighted mean matrix if its entries satisfy

$$
a_{j, k}=\lambda_{k} / \Lambda_{j}, 1 \leq k \leq j ; \quad \Lambda_{j}=\sum_{i=1}^{j} \lambda_{i}, \lambda_{i} \geq 0, \lambda_{1}>0 .
$$

We refer to the $n$-tuple $\left(a_{n, 1}, a_{n, 2}, \cdots, a_{n, n}\right)$ as the $n$-th row of a summability matrix $A$ and then have the following result of Bennett (6], Theorem 1.14) for the $l^{p}$ operator norm of $A$.

Theorem 1.1. Let $p>1$ be fixed and suppose $A$ is a summability matrix. If the rows of $A$ are decreasing, then $\|A\|_{p, p} \geq p /(p-1)$. If the rows of $A$ are increasing, then $\|A\|_{p, p} \leq p /(p-1)$.

The above theorem, when applied to weighted mean matrixes, gives the following inequality ([6], Corollary 4.10).

Theorem 1.2. If $0<\lambda_{1} \leq \lambda_{2} \leq \cdots$ and $0<p<1$, then

$$
\sum_{n=1}^{\infty}\left|\frac{\sum_{i=1}^{n} \lambda_{i} a_{i}^{p}}{\sum_{i=1}^{n} \lambda_{i}}\right|^{1 / p} \leq\left(\frac{1}{1-p}\right)^{1 / p} \sum_{n=1}^{\infty}\left|a_{n}\right| .
$$

Even though the constant in the above theorem is best possible, some improvement may be possible with specific choices of the $\lambda_{i}$ 's. For example, the following two inequalities were claimed to hold by Bennett ([5], pages 40-41; see also [6], page 407):

$$
\begin{aligned}
\sum_{n=1}^{\infty}\left|\frac{1}{n^{\alpha}} \sum_{i=1}^{n}\left(i^{\alpha}-(i-1)^{\alpha}\right) a_{i}\right|^{p} & \leq\left(\frac{\alpha p}{\alpha p-1}\right)^{p} \sum_{n=1}^{\infty}\left|a_{n}\right|^{p}, \\
\sum_{n=1}^{\infty}\left|\frac{1}{\sum_{i=1}^{n} i^{\alpha-1}} \sum_{i=1}^{n} i^{\alpha-1} a_{i}\right|^{p} & \leq\left(\frac{\alpha p}{\alpha p-1}\right)^{p} \sum_{n=1}^{\infty}\left|a_{n}\right|^{p},
\end{aligned}
$$

whenever $\alpha>0, p>1, \alpha p>1$.

We have no access to the proofs of Bennett and, as far as we know, his proofs are not yet published. However, we find that the following unpublished result of J. Cartlidge $[8]$ is very useful to treat the above two inequalities. Here we quote his theorem according to [2] (Theorem C, p. 416):

Theorem 1.3. Let $1<p<\infty$ be fixed. Let $A$ be a weighted mean matrix given by (1.2). If

$$
L=\sup _{n}\left(\frac{\Lambda_{n+1}}{\lambda_{n+1}}-\frac{\Lambda_{n}}{\lambda_{n}}\right)<p
$$

then $\|A\|_{p, p} \leq p /(p-L)$.

We will apply the above theorem to prove (1.3) for $\alpha \geq 1, p>1, \alpha p>1$ and (1.4) for $p>1, \alpha \geq 2, \alpha p>1$ and $p>1,0<\alpha \leq 1, \alpha p>1$ in section 3

Suppose $a_{n} \geq 0$. By a change of variables $a_{n} \rightarrow a_{n}^{1 / p}$ and letting $p \rightarrow \infty$, (1.1) gives the well-known Carleman's inequality:

$$
\sum_{n=1}^{\infty}\left(\prod_{k=1}^{n} a_{k}\right)^{\frac{1}{n}} \leq e \sum_{n=1}^{\infty} a_{n}
$$


We refer the reader to the survey article [14] and the references therein for an account of Carleman's inequality. Among the various generalizations of Carleman's inequality, we mention a result of E. Love [13], who proved that for $\alpha>0, \lambda_{i}=$ $i^{\alpha}-(i-1)^{\alpha}$,

$$
\sum_{n=1}^{\infty}\left(\prod_{i=1}^{n} a_{i}^{i^{\alpha}-(i-1)^{\alpha}}\right)^{1 / n^{\alpha}} \leq e^{\frac{1}{\alpha}} \sum_{n=1}^{\infty} a_{n}
$$

and the constant $e^{\frac{1}{\alpha}}$ is best possible. We note here that after a change of variables $a_{n} \rightarrow a_{n}^{1 / p}$, (1.6) corresponds to the limiting case $p \rightarrow \infty$ of (1.3).

R. Redheffer gave a remarkable proof of Hardy's inequality in [15] by developing the method of "recurrent inequalities". His method also works for Carleman's inequality. Another proof of Carleman's inequality was given by him in [16, and his result has been generalized by $\mathrm{H}$. Alzer 1 and most recently by J. Pečarić and K. Stolarsky [14], who proved for $b_{n}>0, N \geq 1, G_{n}=\left(\prod_{i=1}^{n} a_{i}\right)^{1 / n}$,

$$
\sum_{n=1}^{N} \Lambda_{n}\left(b_{n}-1\right) G_{n}+\Lambda_{N} G_{N} \leq \sum_{n=1}^{N} \lambda_{n} G_{n} b_{n}^{\Lambda_{n} / \lambda_{n}}
$$

In this paper, we will use Redheffer's method to give a weighted version of his treatment of Hardy's and Carleman's inequalities. As we shall see, our result for $1<p<\infty$ is less satisfactory than that of Cartlidge's and the result is almost the same as his for the limiting case.

From now on we will assume $a_{n} \geq 0$ for $n \geq 1$ and any infinite sum converges.

\section{LEMMAS}

Lemma 2.1. If $s \geq 1, n \geq 1$, then

$$
\begin{aligned}
\frac{(n+1)^{s}}{(n+1)^{s}-n^{s}}-\frac{n^{s}}{n^{s}-(n-1)^{s}} & \leq \frac{1}{s}, \\
\frac{s}{s+1} \frac{n^{s}(n+1)^{s}}{(n+1)^{s}-n^{s}} & \leq \sum_{i=1}^{n} i^{s} .
\end{aligned}
$$

Inequality (2.2) reverses when $-1<s \leq 1$.

Proof. This is a result of V. Levin and S. Stečkin, see Lemma 2 on page 18 in [12. Inequality (2.1) is equivalent to inequality (2) in their proof of Lemma 2. They only proved the case $s \geq 0$ for (2.2), but one checks easily that their proof extends to the case $s>-1$.

Lemma 2.2 ([6, Lemma 2.11]). Fix $p>1$. Suppose that $A, B$ are matrices with non-negative entries, and that the rows of $B$ are decreasing, i.e.

$$
b_{j, k} \geq b_{j, k+1}(j, k=1,2, \cdots) .
$$

If

$$
\sum_{k=1}^{n} a_{j, k} \geq \sum_{k=1}^{n} b_{j, k}(j, n=1,2, \cdots),
$$

then $\|A\|_{p, p} \geq\|B\|_{p, p}$. 
Lemma 2.3 ([7, Proposition 5]). For $n \geq 1,0 \leq \alpha<1$,

$$
\left(\frac{\sum_{i=1}^{n} i^{\alpha-1} / n}{\sum_{i=1}^{n+1} i^{\alpha-1} /(n+1)}\right)^{\frac{1}{\alpha-1}} \geq \frac{n+1}{n+2} \geq \frac{n}{n+1} .
$$

Lemma 2.4. Let $\Lambda_{k}=\sum_{i=1}^{k} \lambda_{i}, \lambda_{i}>0$ and $S_{n}=\sum_{i=1}^{n} \lambda_{i} a_{i}$. Let $0 \neq p<1$ be fixed and let $\left(\mu_{i}\right)_{i \geq 1},\left(\eta_{i}\right)_{i \geq 1}$ be two sequences of real numbers such that $\mu_{i} \leq \eta_{i}$ for $0<p<1$ and $\mu_{i} \geq \eta_{i}$ for $p<0$. Then for $n \geq 2$,

$$
\sum_{i=2}^{n-1}\left[\mu_{i}-\left(\mu_{i+1}^{q}-\eta_{i+1}^{q}\right)^{1 / q}\right] S_{i}^{1 / p}+\mu_{n} S_{n}^{1 / p} \leq\left(\mu_{2}^{q}-\eta_{2}^{q}\right)^{1 / q} \lambda_{1}^{1 / p} a_{1}^{1 / p}+\sum_{i=2}^{n} \eta_{i} \lambda_{i}^{1 / p} a_{i}^{1 / p} .
$$

Proof. This is essentially due to R. Redheffer [15. We note for $k \geq 2$,

$$
\mu_{k} S_{k}^{1 / p}-\eta_{k} \lambda_{k}^{1 / p} a_{k}^{1 / p}=S_{k-1}^{1 / p}\left(\mu_{k}(1+t)^{1 / p}-\eta_{k} t^{1 / p}\right) \leq\left(\mu_{k}^{q}-\eta_{k}^{q}\right)^{1 / q} S_{k-1}^{1 / p},
$$

with $t=\lambda_{k} a_{k} / S_{k-1}$ (compare this with the one on page 688 of [15]). The lemma then follows by summing (2.6) for $2 \leq k \leq n$.

Lemma 2.5. Let $\Lambda_{k}=\sum_{i=1}^{k} \lambda_{i}, \lambda_{i}>0$ and $G_{k}=\left(\prod_{i=1}^{k} a_{i}^{\lambda_{i}}\right)^{1 / \Lambda_{k}}$. Then for $\mu_{i}>0, n \geq 2$,

$$
G_{1}+\sum_{i=2}^{n-1}\left(\frac{\Lambda_{i} \mu_{i}}{\lambda_{i}}-\frac{\Lambda_{i}}{\lambda_{i+1}}\right) G_{i}+\frac{\Lambda_{n} \mu_{n}}{\lambda_{n}} G_{n} \leq\left(1+\frac{\Lambda_{1}}{\lambda_{2}}\right) a_{1}+\sum_{i=2}^{n} \mu_{i}^{\frac{\Lambda_{i}}{\lambda i_{i}}} a_{i} .
$$

Proof. This is essentially due to R. Redheffer [15]. We note for $k \geq 2, \mu>0, \eta>0$,

$$
\mu G_{k}-\eta a_{k}=G_{k-1}\left(\mu t-\eta t^{\frac{\Lambda_{k}}{\lambda_{k}}}\right) \leq G_{k-1}\left(\frac{\Lambda_{k-1}}{\lambda_{k}}\right) \eta^{\frac{-\lambda_{k}}{\Lambda_{k-1}}}\left(\frac{\mu \lambda_{k}}{\Lambda_{k}}\right)^{\frac{\Lambda_{k}}{\Lambda_{k-1}}},
$$

where $t^{\frac{\Lambda_{k}}{\lambda_{k}}}=a_{k} / G_{k-1}$ (compare this with the one on page 686 of [15]). By setting $\mu_{k} \Lambda_{k} / \lambda_{k}=\mu, \eta=\mu_{k}^{\Lambda_{k} / \lambda_{k}}$, we get

$$
\frac{\Lambda_{k} \mu_{k}}{\lambda_{k}} G_{k}-a_{k} \mu_{k}^{\frac{\Lambda_{k}}{\lambda_{k}}} \leq \frac{\Lambda_{k-1}}{\lambda_{k}} G_{k-1} .
$$

The lemma then follows by summing (2.7) for $2 \leq k \leq n$ and adding $G_{1}=a_{1}$.

\section{Applications of Cartlidge's Theorem}

We say a weighted mean matrix $A$ given by $(1.2)$ is generated by a logarithmicoexponential function if for all sufficiently large $n, \lambda_{n}:=l(n)$, where $l(x)$ is a positive logarithmico-exponential function, and a logarithmico-exponential function on $\left[x_{0}, \infty\right]$ is defined by Hardy [10] as a real-valued function defined by a finite combination of ordinary algebraic symbols $(\operatorname{viz},+,-, \times, \div, \sqrt[n]{ })$ and the functional symbols $\log (\cdot)$ and $e^{(\cdot)}$, operating on the real variable $x$ and on real constants.

We note first the following theorem of F. Cass and W. Kratz [9]:

Theorem 3.1. Let $1<p<\infty$ be fixed. Let $A$ be a weighted mean matrix given by (1.2), with A generated by a logarithmico-exponential function. Suppose $\lim _{n \rightarrow \infty} \Lambda_{n} / n \lambda_{n}=L<p$. Then $\|A\|_{p, p}<\infty$ if and only if $L<p$, in which case $p /(p-L) \leq\|A\|_{p, p}$. 
It is easy to see $\lim _{n \rightarrow \infty} n^{\alpha-1} /\left(n^{\alpha}-(n-1)^{\alpha}\right)=1 / \alpha$ and the simplest EulerMaclaurin formulae give

$$
\sum_{i=1}^{n} f(i)=\int_{1}^{n} f(x) d x+f(1)+\int_{1}^{n}(x-[x]) f^{\prime}(x) d x,
$$

for $f$ having continuous derivative $f^{\prime}$, where $[x]$ denotes the largest integer not exceeding $x$. It then follows that

$$
\sum_{i=1}^{n} i^{\alpha-1}=n^{\alpha} / \alpha+o\left(n^{\alpha}\right) .
$$

Thus for any fixed $p>1$, thanks to Theorem 3.1, we know (1.3)-1.4 can only hold for $\alpha>0, \alpha p>1$, and if they hold for some $\alpha>0, \alpha p>1$, then the constant $(\alpha p /(\alpha p-1))^{p}$ is best possible.

Now we apply Cartlidge's Theorem to get

Corollary 3.1. Inequality (1.3) holds for $p>1, \alpha \geq 1, \alpha p>1$ and the constant there is best possible.

Proof. Apply Theorem[1.3 with $\lambda_{i}=i^{\alpha}-(i-1)^{\alpha}$. It follows from Lemma 2.1 that $\Lambda_{n+1} / \lambda_{n+1}-\Lambda_{n} / \lambda_{n} \leq 1 / \alpha$, for $n \geq 1, p>1, \alpha \geq 1, \alpha p>1$, and this completes the proof.

We note Corollary 3.1 implies (1.6) for $\alpha \geq 1$. Now if we apply Theorem 1.3 to (1.4), we need to show

$\sum_{i=1}^{n+1} i^{\alpha-1} /(n+1)^{\alpha-1}-\sum_{i=1}^{n} i^{\alpha-1} / n^{\alpha-1}=1+\left(\frac{1}{(n+1)^{\alpha-1}}-\frac{1}{n^{\alpha-1}}\right) \sum_{i=1}^{n} i^{\alpha-1} \leq 1 / \alpha$.

By Lemma 2.1 we get

Corollary 3.2. Inequality (1.4) holds for $p>1, \alpha \geq 2, \alpha p>1$ and $p>1,0<\alpha \leq$ $1, \alpha p>1$ and the constant there is best possible.

We note here (1.5) fails to hold when $0<\alpha<1$ in (1.3) or $1<\alpha<2$ in (1.4) by checking the case $n=1$. We also note if (1.3) holds for $p>1,0<\alpha \leq 1, \alpha p>1$, then (1.4) holds for $p>1,0<\alpha \leq 1, \alpha p>1$ since we can apply Lemma 2.2 here with $A$ the matrix given by (1.3) and $B$ the matrix given by (1.4). It is easy to check that condition (2.3) is satisfied by $B$ and (2.4) follows from Lemma 2.3.

\section{Generalizations of Redheffer's Results}

Theorem 4.1. Assume the same conditions in Lemma 2.4 and let $0<p<1$ be fixed. Suppose there exists a positive constant $c$ such that $c^{-1}+1 \leq c^{-1 / p}$ and

$$
c \leq 1-p+(1-p)\left(\lambda_{i}^{-q}-\lambda_{i-1}^{-q}\right) \Lambda_{i-1} \lambda_{i}^{q / p}, i \geq 2
$$

Then for $0<p<1$,

$$
\sum_{i=1}^{\infty}\left(S_{i} / \Lambda_{i}\right)^{1 / p} \leq c^{-1 / p} \sum_{i=1}^{\infty} a_{i}^{1 / p}
$$


Proof. It suffices to prove the theorem for any integer $n \geq 1$. We note first that condition (4.1) is equivalent to

$$
q^{-1}\left(1-c^{-1}+c^{-1} \Lambda_{i-1} \lambda_{i}^{q / p}\left(\lambda_{i-1}^{-q}-\lambda_{i}^{-q}\right)\right) \geq 1, i \geq 2 .
$$

By setting $\eta_{i}=\lambda_{i}^{-1 / p}, \mu_{i}^{q}=\lambda_{i}^{-q / p}+\Lambda_{i-1} / c \lambda_{i-1}^{q}$ in (2.5), we can rewrite the left-hand side of (2.5) as

$$
\sum_{i=2}^{n-1}\left[\left(\lambda_{i}^{-q / p}+\Lambda_{i-1} / c \lambda_{i-1}^{q}\right)^{1 / q}-\left(\Lambda_{i} / c \lambda_{i}^{q}\right)^{1 / q}\right] S_{i}^{1 / p}+\mu_{n} S_{n}^{1 / p} .
$$

By the mean value theorem,

$$
\begin{aligned}
\left(\lambda_{i}^{-q / p}+\Lambda_{i-1} /\right. & \left.c \lambda_{i-1}^{q}\right)^{1 / q}-\left(\Lambda_{i} / c \lambda_{i}^{q}\right)^{1 / q} \\
& \geq q^{-1}\left(\lambda_{i}^{-q / p}+\Lambda_{i-1} / c \lambda_{i-1}^{q}-\Lambda_{i} / c \lambda_{i}^{q}\right)\left(\Lambda_{i} / c \lambda_{i}^{q}\right)^{-1 / p} \\
& =q^{-1}\left(1-c^{-1}+c^{-1} \Lambda_{i-1} \lambda_{i}^{q / p}\left(\lambda_{i-1}^{-q}-\lambda_{i}^{-q}\right)\right)\left(\Lambda_{i} / c\right)^{-1 / p} \\
& \geq\left(\Lambda_{i} / c\right)^{-1 / p} .
\end{aligned}
$$

Here the last inequality follows from (4.3). Thus (2.5) becomes

$$
\sum_{i=1}^{n}\left(S_{i} / \Lambda_{i}\right)^{1 / p} \leq\left(c^{-1}+1\right) a_{1}^{1 / p}+c^{-1 / p} \sum_{i=2}^{n} a_{i}^{1 / p} \leq c^{-1 / p} \sum_{i=1}^{n} a_{i}^{1 / p} .
$$

This completes the proof.

We note here if $0<\lambda_{1} \leq \lambda_{2} \leq \cdots$, we can take $c=1-p$ in (4.1) and one checks easily for $0<p<1,(1-p)^{-1}+1<(1-p)^{-1 / p}$. Theorem 4.1 then implies Theorem 1.2,

We also note the constant given by the above theorem may be less satisfactory. For example the case $\alpha=2, p=2$ in (1.3) corresponds to the case $\lambda_{i}=2 i-1, p=$ $1 / 2, c=3 / 4$ in (4.2). However, direct calculation shows (4.1) is not satisfied in this case. Of course one may try to prove directly

$$
\left(\lambda_{i}^{-q / p}+\Lambda_{i-1} / c \lambda_{i-1}^{q}\right)^{1 / q}-\left(\Lambda_{i} / c \lambda_{i}^{q}\right)^{1 / q} \geq\left(\Lambda_{i} / c\right)^{-1 / p} .
$$

But one checks this fails for $i=2$.

Similarly, the case $\alpha=2, p=2$ in (1.4) corresponds to the case $\lambda_{i}=i, p=$ $1 / 2, c=3 / 4$ in (4.2). One checks in this case that (4.1) holds for $i \geq 2$. However, $c^{-1}+1=7 / 3>16 / 9=c^{-2}$, so the coefficient of $a_{1}$ is slightly larger.

Now we focus our attention on Carleman-type inequalities.

Theorem 4.2. Assume the same conditions in Lemma 2.5, and let $f(x)$ be a real-valued function defined for $x \geq 2$ such that $f(n)=\Lambda_{n} / \lambda_{n}$ for $n \geq 2$ and $0 \leq f(x+1)-f(x) \leq 1 / \alpha$ for some $\alpha>0$. If $\left(1+\frac{\Lambda_{1}}{\lambda_{2}}\right) \leq e^{1 / \alpha}$ for the same $\alpha$, then

$$
\sum_{n=1}^{\infty}\left(\prod_{i=1}^{n} a_{i}^{\lambda_{i}}\right)^{1 / \Lambda_{n}} \leq\left(1+\frac{\Lambda_{1}}{\lambda_{2}}\right) a_{1}+\sum_{i=2}^{n} a_{i}\left(1+\frac{f(i+1)-f(i)}{f(i)}\right)^{f(i)} \leq e^{1 / \alpha} \sum_{n=1}^{\infty} a_{n} .
$$


Proof. It suffices to prove the theorem for any integer $n \geq 2$. Setting $\mu_{i}=$ $f(i+1) / f(i)$ in Lemma 2.5 we get

$$
\begin{aligned}
\sum_{i=1}^{n} G_{i} \leq \sum_{i=1}^{n-1} G_{i}+f(n+1) G_{N} & \leq\left(1+\frac{\Lambda_{1}}{\lambda_{2}}\right) a_{1}+\sum_{i=2}^{n} a_{i}\left(1+\frac{f(i+1)-f(i)}{f(i)}\right)^{f(i)} \\
& \leq e^{1 / \alpha} \sum_{n=1}^{\infty} a_{n}
\end{aligned}
$$

by the conditions of the theorem, and this completes the proof.

Apply Theorem 4.2 to $\lambda_{1}=1, \lambda_{i}=\alpha^{i-1}-\alpha^{i-2}, i \geq 2$ for some $\alpha>1$. Then $f(x)=\alpha /(\alpha-1)$ and we get

Corollary 4.1. For $\alpha>1$,

$$
\sum_{n=1}^{\infty}\left(a_{1} \prod_{k=2}^{n} a_{k}^{\alpha^{k-1}-\alpha^{k-2}}\right)^{1 / \alpha^{n-1}} \leq\left(1+\frac{1}{\alpha-1}\right) a_{1}+\sum_{n=2}^{\infty} a_{n}
$$

Apply Theorem 4.2 to $\lambda_{i}=\alpha^{i}, i \geq 1$ for some $\alpha>0$. Then $f(i+1)-f(i)=\alpha^{-i}$ and we get

Corollary 4.2. For $\alpha>0$,

$$
\sum_{n=1}^{\infty}\left(\prod_{k=1}^{n} a_{k}^{\alpha^{k-1}}\right)^{\left(\alpha^{n}-1\right) /(\alpha-1)} \leq\left(1+\frac{1}{\alpha}\right) a_{1}+\sum_{n=2}^{\infty} e^{1 / \alpha^{n}} a_{n} \leq \sum_{n=1}^{\infty} e^{1 / \alpha^{n}} a_{n} .
$$

We end the paper by noting that if we take $\lambda_{i}=(i(i+1))^{-1}$ in Theorem 4.2 then $f(x)=x^{2}$ and we get back a result of Redheffer (see [15], page 693):

\section{Corollary 4.3.}

$$
\sum_{n=1}^{\infty}\left(\prod_{k=1}^{n} a^{1 / k(k+1)}\right)^{(n+1) / n} \leq \sum_{n=1}^{\infty} e^{2 n} a_{n}
$$

\section{REFERENCES}

[1] H. Alzer, On Carleman's inequality, Portugal. Math., 50 (1993), 331-334. MR1244510 (94j:26030)

[2] G. Bennett, Some elementary inequalities, Quart. J. Math. Oxford Ser. (2), 38 (1987), 401425. MR0916225 (88k:26018)

[3] G. Bennett, Some elementary inequalities, II, Quart. J. Math. Oxford Ser. (2), 39 (1988), 385-400. MR0975904 (90a:26030)

[4] G. Bennett, Some elementary inequalities, III, Quart. J. Math. Oxford Ser. (2), 42 (1991), 149-174. MR1107279 (92h:26021)

[5] G. Bennett, Factorizing the classical inequalities, Mem. Amer. Math. Soc., 120 (1996), 1-130. MR.1317938(96h:26020)

[6] G. Bennett, Inequalities complimentary to Hardy, Quart. J. Math. Oxford Ser. (2), 49 (1998), 395-432. MR 1652236 (2000a:26018)

[7] G. Bennett and G. Jameson, Monotonic averages of convex functions, J. Math. Anal. Appl., 252 (2000), 410-430. MR1797865 (2001k:26013)

[8] J. M. Cartlidge, Weighted mean matrices as operators on $l^{p}$, Ph.D. thesis, Indiana University, 1978.

[9] F. P. Cass and W. Kratz, Nörlund and weighted mean matrices as operators on $l_{p}$, Rocky Mountain J. Math. 20 (1990), 59-74. MR1057975 (91g:47024)

[10] G. H. Hardy, Orders of Infinity, Cambridge Univ. Press, 1954. MR0349922 (50:2415)

[11] G. H. Hardy, J.E. Littlewood and G. Pólya, Inequalities, Cambridge Univ. Press, 1952. MR $0046395(13: 727 \mathrm{e})$ 
[12] V. I. Levin and S.B. Stečkin, Inequalities, Amer. Math. Soc. Transl. (2), 14 (1960), 1-29. MR0112925 (22:3771)

[13] E. Love, Inequalities related to Carleman's inequality, Inequalities (Birmingham, 1987), 135141, Lecture Notes in Pure and Applied Mathematics vol. 129, Dekker, New York, 1991. MR 1112576 (92i:26016)

[14] J. Pečarić and K. Stolarsky, Carleman's inequality: history and new generalizations, Aequationes Math., 61 (2001), 49-62. MR1820809 (2002m:26029)

[15] R. M. Redheffer, Recurrent inequalities, Proc. London Math. Soc. (3), 17 (1967), 683-699. MR 0218513 (36:1599)

[16] R. M. Redheffer, Easy proofs of hard inequalities, General inequalities 3 (ed. E. F. Beckenbach and W. Walter), 123-140, Birkhäuser Verlag, Basel, 1983. MR0785770 (86i:26016)

Department of Mathematics, University of Michigan, Ann Arbor, Michigan 48109

E-mail address: penggao@umich.edu 